\title{
ADVANCED IMAGING SYSTEMS CURRICULA AT EPFL
}

\author{
Touradj Ebrahimi and Murat Kunt \\ Signal Processing Laboratory \\ Swiss Federal Institute of Technology at Lausanne - EPFL \\ CH-1015 Lausanne, Switzerland \\ Email: ebrahimi@epfl.ch
}

\begin{abstract}
This paper describes the current status of the image system engineering curriculum at the Swiss Federal Institute of Technology at Lausanne (Ecole Polytechnique Fédérale de Lausanne - EPFL). The responsibility of this curriculum is with the Signal Processing Laboratory of EPFL.
\end{abstract}

\section{GENERAL PRESENTATION}

The Signal Processing Laboratory (LTS) of EPFL is composed of three main areas of research and teaching, key worded as Digital TV, Image analysis and 1-D signal Processing. Digital TV research includes Video processing, Compression, Image quality assessment, Motion estimation and Multimedia systems to name the most important sub-areas. They represent $60 \%$ of the total effort. Image analysis research includes Image sequence analysis for surveillance and automatic recognition, Motion modeling, Database search and Pattern recognition for $20 \%$ of the total effort. The remaining share for $1-D$ signals are related to biomedicine and industrial control and surveillance. The Laboratory hosts an average of 6 visiting scientists or students per year from all over the world and from various backgrounds.

The image system engineering curriculum is based on courses, laboratory sessions on computers, term and diploma projects as well as Ph.D. thesis. All the undergraduate projects are related to ongoing research projects at the $\mathrm{Ph}$. D. level. This connection is very strong in the sense that all the topics for these undergraduate projects correspond to a specific problem of the main research projects. This way of doing brings the undergraduate student to a direct contact with the on going research in the Laboratory. It gives him the earliest possible opportunity to be involved in this research. The Ph.D. student who is the direct supervisor enlarges his experience by the results of the project and reflects it to higher supervisors. Both, the undergraduate student and the Ph.D. student, receive strong motivation by being involved in research. The a priori unknown solution of the project discourages mediocre students to select our projects. Project topics cover a rather wide range of image system engineering applications. They may be improvements of some components or alternative methods for some selected parts of a complex system. They are implemented by simulation on mainly workstations, shared with other users. It is worth mentioning that due to the large amount of time that students spend on these practical projects (210 hours term project and 4 months diploma project) they are immediately operational upon their recruitment in the industry as exemplified by numerous cases of our past students.

The Laboratory organized twice a graduate program on Information Processing with 625 hours teaching and 6 months master equivalent thesis. Most of the program was centered around image processing and analysis going all the way from theory to specific VLSI design. Hands-on laboratory sessions were also included. A total of 30 students graduated from theses programs. We plan to reiterate this program in the future if funds become available.

From the early 80 's, the Laboratory has a unique experience in developing interactive teaching software. This has been done in the Digital Signal Processing (DSP) area, using interactively engineers from the European Nuclear Research Center (CERN) as students. The resulting software evolved into a very efficient and highly user-friendly modern version. Practicing DSP became thus as entertaining as video games or Nintendo plays. We are planning to use our experience to expand this package to image and image sequence processing.

Currently a platform is under development to facilitate the simulations so that various components of a complex image processing system can be programmed in the easiest way similar to plug-in modules of a tool box in a portable, flexible and user-friendly environment. This way, not only the efficiency of the 
existing situation will improve, but also new possibilities for a larger number of students and young researchers will be available. New research topics the Laboratory would like to develop under these conditions are contributions to the new standard MPEG4 for audio-video compression, a new and always optimal way of image compression called dynamic coding, specific medical image compression and processing, etc.

\section{CURRENT IMAGE SYSTEMS ENGINEERING CURRICULUM STATUS}

The Signal Processing Laboratory of EPFL is responsible for the image systems engineering curriculum at EPFL. The Laboratory is one of the largest at EPFL comprising 2 professors (one half time), 4 senior group leaders, 1 system engineer, 1 technician, 3 secretaries ( 2 of them part time) and 28 young researchers ( $\mathrm{Ph} . \mathrm{D}$. students). All the Ph.D. students are supported by external grants from industry, governmental agencies or European projects. The amount corresponding to theses supports exceeds 3.5 million SFr. per year.

The Laboratory has three main activities intimately related : teaching, research and development. Teaching at the undergraduate level corresponds to three courses given in the class room and to term and diploma projects related to the research activities of the Laboratory. The courses are basic digital signal processing, advanced digital signal processing and image processing, totaling to 120 hours teaching in class and 30 hours of class room exercises.

These courses are reinforced by elective laboratory sessions and term and diploma projects. The main teaching policy of the Institute is first to give to the students the basic knowledge in classes, then to let them work on practical problems to complete their education and practical expertise. Laboratory sessions covers 60 hours in the curriculum and are based on computer aided teaching program developed by ourselves. Each student has access to a workstation and runs interactive and highly user-friendly programs to design, implement and practice signal and image processing systems for various applications. A number of systems and applications are suggested but they are also required to imagine their own.

Projects (term and diploma) are designed and implemented in a very specific way. Each Ph.D. student is under the supervision of a group leader and the Director of the Laboratory. The thesis topics in signal and image processing areas are such that, quite regularly in the progress of the work, decisions should be made about one method or another to be used. These decisions are taken mainly on the basis of our experience, intuition and vision. The methods that are not considered may be useful as well since we can never be sure, a priori, of the goodness of our decisions. These un-retained methods are proposed to students as term projects under the direct supervision of the corresponding Ph.D. student who acts as teaching assistant. This way of doing has many advantages. It brings the undergraduate student to a direct contact with the on going research in the Laboratory. It gives him the earliest possible opportunity to be involved in this research. The Ph.D. student enlarge his experience by the results of the project and reflects it to higher supervisors. Both, the undergraduate student and the Ph.D. student, receive strong motivation by being involved in research. The a priori unknown solution of the project discourage mediocre students to select our projects.

Term projects run over the entire semester, both winter and summer, totaling to 105 hours for each project per term. Their topic is related to ongoing research of the Laboratory and collaborations of LTS in European projects or international consortia. In particular, they consist in improvement or implementation of ideas which are to be integrated as a component into an already existing system. In addition to direct interaction between the student and his supervisor, regular progress meetings to senior researchers and the Director of the Laboratory are organized on a monthly basis. At the end of a such project, the student has a good understanding of a state-of-the-art technique in digital signal or image processing, both from a theoretical and practical points of view. An oral presentation as well as a written report is required from the students at the end of their projects. These reports are filed in the Library of the Laboratory for later use. The software produced by the students is also integrated into appropriate software libraries if they are well documented and properly coded.

Diploma projects run full time over 4 months. Each project corresponds to a given signal and image processing problem with application. Every graduate student of the Laboratory is required to produce an original research work prior to the obtention of his/her engineering diploma. A diploma project is longer in duration and in a more advanced basis than a semester project. The framework however resembles that of semester projects. The student is supervised by a researcher involved in the topic. More frequent progress sessions with senior researchers and the Director of the Laboratory are organized along with an intermediate oral presentation. The last step of the project is a final report and an oral presentation of the major achievements of the diploma work. Again, these reports are filed in the Library of the Laboratory for later use. 
The software produced by the graduating students is much more reusable than that produced during term projects due to the programming experience gained. It is integrated into appropriate software libraries of the Laboratory.

It is also worth mentioning that the Laboratory is involved in a number of international networks of exchange, in which researchers, namely, invited professors, postdoctoral fellows, and exchange students, spend some time in the Laboratory (minimum 3 months, maximum 1 year), either to reinforce an existing project or to prepare grounds for a new one. The number of such visitors is around an average of 6 per year. It is also the Laboratory's policy to play an active role within Switzerland and within Europe through participation to a large number of national and European programs (COST, EUREKA, ESPRIT R\&D, ESPRIT BRA, RACE, HCM, ACTS, IT, TMR). Over the last 10 years, the Laboratory participated to more than 21 such projects, establishing strong connections to various other swiss and international universities and companies. Furthermore, the Laboratory plays a central role in the management of the Eurcom Institute, cofounded by EPFL and France Télécom in Sophia-Antipolis, France, specialized in communication system engineering. Image processing there is one of the three major areas.

The coverage of various topics are directly related to the size and the number of research projects of the Laboratory. As a first classification, we can say that $60 \%$ of the topics are related to Digital Television, Video processing, Compression, Image quality assessment, Motion estimation and Multimedia systems. Image sequence analysis, Motion modeling, Database search and pattern recognition correspond to $20 \%$. The remaining $20 \%$ is covered by one dimensional signal processing problems.

Given the emphasis in LTS on team work, and interproject exchange, a major topic is that of an efficient, simple and rapid portability of materials developed by various projects and among different researchers. The Signal Processing Laboratory has therefore started the development of an object oriented programming environment (in $\mathrm{C}++$ ) defining a number of rules and conventions in order to allow a virtually automatic reuse of source codes produced for specific tasks for a given image engineering system. In this view, all results in terms of working systems of sub-systems are documented and have been gathered in a central software pool, using the latest shared software development tools.

Our latest student projects as well as the implementation of our current topics of interest (international project and industrial contracts) have been made un- der the above structure. The first results of a such experiment have been very successful and allowed a great time saving for the reuse of other software, interfacing and debugging process, as well as more efficient working systems.

\section{CURRICULUM GROWTH}

The early success in the use of a software platform for implementation of image engineering systems has encouraged its development to a more advanced phase, with an improved and a more complete library of tools and systems.

Beside the continuation of our current research, as well as the completion of international projects and industrial contract, future work is oriented to giving input in new projects such as MPEG-4, dynamic coding, medical imaging, and so on. The following topics of research, from which a large number of new student projects at all levels will be deduced, are our focus of interest in the following years:

\subsection{Syntax oriented coding:}

In a wide range of applications, including multimedia, the general tendency in future product developments will be in the direction of programmable rather than hardwired equipments. This is further encouraged by the increasing processing power available from new generations of CPUs and DSPs. The idea behind this approach is to precede a compressed data with the syntax description of the decompression algorithm (or equivalent indications) which will be programmed in the DSP or CPU. This way, a number of drawbacks will be removed in the current systems. To name a few one can mention: the problem of compatibility, standardization burden, and possibility of continuous improvements.

\subsection{Video compression for distributed multi- media applications:}

A number of multimedia services require an access to data (text, audio, and video) stored in various servers distributed in different locations. In order to reduce the traffic on the network, as well as the waiting time delay for a given request, it is mandatory to use compression prior to data transmission. The idea of this work is to use a double compression scheme. The first would serve to reduce the bitrate as much as possible, without any restrictions such as browsing. It will used for transmission from the remote host to local host. A second compression method is then used to recompress the data in the local host with less performances in 
terms of compression but allowing functionalities such as browsing.

\subsection{Region based coding with novel functiona- lities:}

New functionalities are needed in a number of interactive applications in multimedia which require compression. Most of these functionalities consist in manipulation of different objects. If the notion of object is already present in the compressed bit stream, it is obviously simpler and more efficient to search for given objects and to perform the necessary manipulations directly. It is therefore important to investigate region based techniques allowing object manipulations in a direct form. As an example, it would be possible to enhance the resolution and clarity of a given region of the image at the request of the user.

\subsection{Dynamic coding:}

It is well known that video data is a highly non stationaryprocess. In multimedia applications all sort of images could be transmitted between terminals. Among all the techniques already investigated in the literature, some perform better in particular regions of an image better than others. Typically, subband/wavelet schemes are knows to perform well in areas with textures, whereas techniques based on segmentation, or morphological operators perform well at areas with sharp edges and contours. Similarly, methods using linear transforms produce poor results in areas with text or graphics. Dynamic coding is a solution to solve the drawbacks existing in a given scheme, while still maintaining its strong performance where appropriate. The basic idea behind dynamic coding is simple yet very powerful. The image sequence is divided into several regions with uniform characteristics. Every region is encoded using a multitude of compression techniques. Among all these techniques, the one which is the most efficient is chosen and the compressed bitstream of the region using the best coding technique is sent to the decoder along with information informing which technique was chosen for its coding.

\section{CURRENT OBJECTIVES}

The major objectives are to complete the implementation of various tools in the $\mathrm{C}++$ platform in order to produce operational systems showing the feasibility of our research activities in "dynamic coding". The dynamic coding will be applied to two main applications, namely, the MPEG-4 standardization activity, and medical imaging in a multimedia environment.
The basic structure in our approach is to view the intended image engineering systems as algorithms conceived for specific applications. Each algorithm in turn is a set of basic tools that are put together according to a given strategy. Under this perspective, a particular system will be an algorithm fulfilling the requirements of a given application, by means of appropriate tools.

The applications under consideration are:

- Very low bitrate video coding in multimedia environment

- Lossless and nearly lossless coding of medical images

Both applications will allow object manipulation functionalities. Dynamic coding algorithm will be used in order to realize working systems for these applications. The following tools are being developed in this context:

- Region interior coding

- Region shape coding

- Region oriented motion estimation

- Segmentation

- Coding mode decision

- Object tracking

- Rate controller

Each of the above tools, as well as part of the algorithms are developed in the framework of term and diploma projects.

\section{CONCLUSION}

This paper presented the imaging system curricula in the Signal Processing Laboratory of EPFL. The main aspects of the curricula were outlined, namely, teaching, research, and development activities. Special care is given to establish the correct compromise when dividing our efforts among each of these highly independent axes.

More information about activities of the imaging system curricula at EPFL can be found online at the Web server "http//ltswww.epfl.ch/". 